\title{
PERTURBATIONS OF CAUSTICS AND FRONTS
}

\author{
GOO ISHIKAWA \\ Department of Mathematics, Hokkaido University \\ Sapporo 060-0810, Japan \\ E-mail: ishikawa@math.sci.hokudai.ac.jp
}

Dedicated to Professor Tatsuo Suwa for his 60th birthday

1. Introduction. A degenerate singularity of a plane caustic bifurcates into several cusps. Then we may ask:

How many cusps do there appear after a stable perturbation?

In this article, we review the algebraic formula for the number of complex cusps, thus the upper estimate of the number of real cusps, and, gradually, we show their several possible, known and unknown, generalisations, in a comprehensible manner, as best as possible.

We start with the ordinary singularity theory. Let $f:\left(\mathbf{C}^{2}, 0\right) \rightarrow\left(\mathbf{C}^{2}, 0\right)$ be an unstable holomorphic map-germ. We consider the problem of counting the number of cusps after a stable perturbation. We note that the cusps appear, for the perturbed $f$, at a point on the singular locus $J=0$, defined by the Jacobian $J=J\left(f_{1}, f_{2}\right)$, where the extended mapping $\left(J, f_{1} \cdot f_{2}\right): \mathbf{C}^{2} \rightarrow \mathbf{C}^{3}$ is not immersive. So we count the intersection number at 0 of the jet section $j^{2} f: \mathbf{C}^{2} \rightarrow J^{2}\left(\mathbf{C}^{2}, \mathbf{C}^{2}\right)$ with the variety in $J^{2}\left(\mathbf{C}^{2}, \mathbf{C}^{2}\right)$ defined by $J$ and 2 -minors of the Jacobi matrix of $\left(J, f_{1}, f_{2}\right)$. Since $j^{2} f$ is an immersion, setting the ideal

$$
\mathcal{J}=\mathcal{J}_{1,1}=\left\langle J\left(f_{1}, f_{2}\right), J\left(f_{1}, J\left(f_{1}, f_{2}\right)\right), J\left(f_{2}, J\left(f_{1}, f_{2}\right)\right)\right\rangle_{\mathcal{O}_{2}},
$$

we have:

ThEOREM 1.1 ([3], see also [8]). The number $\kappa$ of cusps for a stable perturbation $\tilde{f}$ from $f:\left(\mathbf{C}^{2}, 0\right) \rightarrow\left(\mathbf{C}^{2}, 0\right)$ is equal to the dimension over $\mathbf{C}$ of the quotient algebra (doubly iterated Jacobian algebra) $Q=\mathcal{O}_{2} / \mathcal{J}$ :

$$
\kappa=\operatorname{dim}_{\mathbf{C}} \mathcal{O}_{2} /\left\langle J\left(f_{1}, f_{2}\right), J\left(f_{1}, J\left(f_{1}, f_{2}\right)\right), J\left(f_{2}, J\left(f_{1}, f_{2}\right)\right)\right\rangle_{\mathcal{O}_{2}},
$$

provided $\operatorname{dim}_{\mathbf{C}} Q<\infty$.

2000 Mathematics Subject Classification: Primary 58K60; Secondary 13C14, 53D12.

Financial support from Grant-in-Aid for Scientific Research, No. 14340020.

The paper is in final form and no version of it will be published elsewhere. 
Second, we consider the problem on caustics. Let $f:\left(\mathbf{C}^{2}, 0\right) \rightarrow T^{*} \mathbf{C}^{2}=\mathbf{C}^{4}$ be a Lagrangian immersion. Recall the cotangent bundle $T^{*} \mathbf{C}^{2}$ with the canonical coordinates $\left(p_{1}, p_{2} ; q_{1}, q_{2}\right)$ has the symplectic 2 -form $\omega=d p_{1} \wedge d q_{1}+d p_{2} \wedge d q_{2}$ and the canonical Lagrangian projection $\pi: T^{*} \mathbf{C}^{2} \rightarrow \mathbf{C}^{2}, \pi(p, q)=q$. We suppose the immersion $f$ satisfies $f^{*} \omega=0$. Then $\pi \circ f$ is called the Lagrangian mapping and its critical value set is called caustic of $f$. Now assume $f$ is not necessarily Lagrange stable with respect to $\pi$, and ask:

How many cusps do there appear after a Lagrange stable perturbation?

We denote by $\kappa=\kappa(\pi \circ \tilde{f})$ the number of cusps near the origin, under the projection $\pi$, of a Lagrange stable perturbation $\tilde{f}$ of $f$. Since $\pi \circ \tilde{f}$ is also a stable perturbation of $\pi \circ f$, we can calculate the number of cusps by the above theorem from $\pi \circ f:\left(\mathbf{C}^{2}, 0\right) \rightarrow\left(\mathbf{C}^{2}, 0\right)$.

In Lagrangian singularity theory, Lagrangian immersions and their caustics are analysed via their generating families [1]. Thus we are also led to the following question:

What is the algebraic formula for $\kappa$ in term of the generating family of $f$ ?

Let $F:\left(\mathbf{C}^{r} \times \mathbf{C}^{2}, 0\right) \rightarrow \mathbf{C}$ be a Morse family. (It is not assumed to be $\mathcal{R}^{+}$-stable [1].) That $F$ is a Morse family means that the catastrophe set

$$
C(F):=\left\{(x, \lambda) \in \mathbf{C}^{r} \times \mathbf{C}^{2} \mid \frac{\partial F}{\partial x_{1}}=0, \ldots, \frac{\partial F}{\partial x_{r}}=0\right\}
$$

is regularly defined in $\mathbf{C}^{r} \times \mathbf{C}^{2}$, namely, $\left(\frac{\partial F}{\partial x_{1}}, \ldots, \frac{\partial F}{\partial x_{r}}\right):\left(\mathbf{C}^{r} \times \mathbf{C}^{2}, 0\right) \rightarrow \mathbf{C}^{r}$ is submersive along $C(F)$. Then the mapping $f=L(F): C(F) \rightarrow T^{*} \mathbf{C}^{2}$ defined by

$$
L(F)(x, \lambda)=\left(\frac{\partial F}{\partial \lambda_{1}}, \frac{\partial F}{\partial \lambda_{2}}, \lambda_{1}, \lambda_{2}\right),
$$

is a Lagrangian immersion. Conversely, any Lagrangian immersion can be constructed by this method, up to parametrisation. Thus we call $F$ the generating family of the Lagrangian immersion $f$. The singularities of caustics of $f$ coincides with the singularities of the projection $\left.\Pi\right|_{C(F)}: C(F) \rightarrow \mathbf{C}^{2}$, where $\Pi: \mathbf{C}^{r} \times \mathbf{C}^{2} \rightarrow \mathbf{C}^{2}$ is the projection $(x, \lambda) \mapsto \lambda$. The critical value set of $\left.\Pi\right|_{C(F)}$ is called the bifurcation set of $F$, which agrees with the caustic of the Lagrangian immersion $L(F)$.

Now we set the Hessian of $F$ by

$$
J(\nabla F):=J\left(\frac{\partial F}{\partial x_{1}}, \ldots, \frac{\partial F}{\partial x_{r}}\right)=\frac{\partial\left(\frac{\partial F}{\partial x_{1}}, \ldots, \frac{\partial F}{\partial x_{r}}\right)}{\partial\left(x_{1}, \ldots, x_{r}\right)},
$$

denoting by $J\left(a_{1}, \ldots, a_{r}\right)$ the Jacobian $\operatorname{det}\left(\frac{\partial a_{i}}{\partial x_{j}}\right)$ of $a_{1}, \ldots, a_{r}$ by $x_{1}, \ldots, x_{r}$, and the "secondary Hessian" of $F$ by

$$
J_{i}^{(2)}(\nabla F):=J\left(\frac{\partial F}{\partial x_{1}}, \ldots, \frac{\partial F}{\partial x_{i-1}}, J(\nabla F), \frac{\partial F}{\partial x_{i+1}}, \ldots, \frac{\partial F}{\partial x_{r}}\right),
$$

replacing $\frac{\partial F}{\partial x_{i}}$ by $J(\nabla F)$ for $i=1, \ldots, r$. Then we set

$$
\mathcal{J}_{r, 1,1}:=\left\langle\frac{\partial F}{\partial x_{1}}, \ldots, \frac{\partial F}{\partial x_{r}}, J(\nabla F), J_{1}^{(2)}(\nabla F), \ldots, J_{r}^{(2)}(\nabla F)\right\rangle_{\mathcal{O}_{r+2}} .
$$

Then we have:

TheOREM 1.2. The number $\kappa$ of cusps of a Lagrange stable perturbation of $L(F)$ is equal to the dimension $\operatorname{dim}_{\mathbf{C}} Q$ of the doubly iterated Jacobian algebra

$$
Q:=\mathcal{O}_{r+2} / \mathcal{J}_{r, 1,1},
$$


provided $\operatorname{dim}_{\mathbf{C}} Q<\infty$.

Note that if $\operatorname{dim}_{\mathbf{C}} Q<\infty$ then $\operatorname{dim}_{\mathbf{C}} \mathcal{O}_{r} /\left\langle\partial \phi / \partial x_{1}, \ldots, \partial \phi / \partial x_{r}\right\rangle<\infty$ for $\phi=F(x, 0)$. Thus $\phi$ has an $\mathcal{R}^{+}$-versal deformation and $F$ is induced from it.

EXAmple 1.3. Set $F\left(x_{1}, \lambda_{1}, \lambda_{2}\right)=x_{1}^{5}+\lambda_{1} x_{1}^{2}+\lambda_{2} x_{1}$, with $r=1$. Then we have $\frac{\partial F}{\partial x_{1}}=5 x_{1}^{4}+2 \lambda_{1} x_{1}+\lambda_{2}, J(\nabla F)=\frac{\partial^{2} F}{\partial x_{1}^{2}}=20 x_{1}^{3}+2 \lambda_{1}$, and $J_{1}^{(2)}(\nabla F)=\frac{\partial^{3} F}{\partial x_{1}^{3}}=60 x_{1}^{2}$. Thus $Q=\mathcal{O}_{3} /\left\langle x_{1}^{2}, \lambda_{1}, \lambda_{2}\right\rangle$. Therefore we have $\kappa=\operatorname{dim}_{\mathbf{C}} Q=2$.

We give here the proof of Theorem 1.2 shortly, since the proof provides the prototype for the proofs of possible generalisations.

Proof of Theorem 1.2. $C(F)$ is defined by $\nabla F=\left(\frac{\partial F}{\partial x_{1}}, \ldots, \frac{\partial F}{\partial x_{r}}\right)=0$ and the singular locus of $\left.\Pi\right|_{C(F)}$ coincides with the singular locus of $\left(\nabla F, \lambda_{1}, \lambda_{2}\right): \mathbf{C}^{r+2} \rightarrow \mathbf{C}^{r+2}$ on $C(F)$, and it is defined by $J(\nabla F)=0$ on $C(F)$. Moreover the cusp locus coincides with the singular locus of $\left(\nabla F, J(\nabla F), \lambda_{1}, \lambda_{2}\right): \mathbf{C}^{r+2} \rightarrow \mathbf{C}^{r+3}$. The $(r+2)$-minors of its Jacobi matrix are

$$
J(\nabla F), J_{1}^{(2)}(\nabla F), \ldots, J_{r}^{(2)}(\nabla F) .
$$

Now consider the jet space $J^{3}\left(\mathbf{C}^{r+2}, \mathbf{C}\right)$. Then jets of Morse families form an open subspace $M \subset J^{3}\left(\mathbf{C}^{r+2}, \mathbf{C}\right)$. We set $C:=\left\{j^{3} F\left(x_{0}\right) \in M \mid \nabla F\left(x_{0}\right)=0\right\}$. Then $C$ is a submanifold of $M$ of codimension $r$. Now the $(r+2)$-minors of Jacobi matrix of $\left(\nabla F, J(\nabla F), \lambda_{1}, \lambda_{2}\right)$, which is considered as an $(r+2) \times(r+3)$ matrix with entries in $\mathcal{O}_{C}$, defines a determinantal variety $\Sigma \subset C$ of codimension 2 . Then $\Sigma$ is CohenMacaulay. Moreover the defining ideal of $\Sigma$ is reduced over the regular locus of $\Sigma$. Then the intersection index of $j^{2} F: \mathbf{C}^{r+2} \rightarrow M$ with $\Sigma$ gives the number of cusps after an $\mathcal{R}^{+}$-stable perturbation of $F$, and it is given by $\operatorname{dim}_{\mathbf{C}} Q$ via $\left(j^{3} F\right)^{*}: \mathcal{O}_{r+2} \leftarrow \mathcal{O}_{M}$.

Here we have two points for the idea of the proof: First we note that, for instance, $J_{i}^{(2)}(\nabla F)$ is expressed by a unique (universal) polynomial on partial derivatives of order not greater than 3 of $F$, for any $F$. In fact, we use throughout this paper the following well-known fact:

LEMMA 1.4. Let $h$ be a polynomial function on $J^{r}\left(\mathbf{C}^{m}, \mathbf{C}^{p}\right)$. Then, for each $i$ from $\{1, \ldots, m\}$, there exists a unique polynomial $\frac{d}{d x_{i}} h$ over $J^{r+1}\left(\mathbf{C}^{m}, \mathbf{C}^{p}\right)$ such that $\left(\frac{d}{d x_{i}} h\right) \circ$ $j^{r+1} f=\frac{\partial}{\partial x_{i}}\left(h \circ j^{r} f\right)$, for any $f:\left(\mathbf{C}^{m}, x_{0}\right) \rightarrow\left(\mathbf{C}^{p}, y_{0}\right), x_{0} \in \mathbf{C}^{m}, y_{0} \in \mathbf{C}^{p}$. The operator $h \mapsto \frac{d}{d x_{i}} h$ is characterised by the properties $\frac{d}{d x_{i}}(h k)=\left(\frac{d}{d x_{i}} h\right) k+h\left(\frac{d}{d x_{i}} k\right)$, and $\frac{d}{d x_{i}} x_{j}=\delta_{i j}, \frac{d}{d x_{i}}\left(y_{\alpha, j}\right)=y_{\alpha+e_{i}, j}$, where $y_{\alpha, j}\left(j^{r} f\left(x_{0}\right)\right)=\frac{\partial^{|\alpha|} y_{j} \circ f}{\partial x^{\alpha}}\left(x_{0}\right)$, and $e_{i}$ equals $(0, \ldots, 1, \ldots 0)$ (i-th component).

Second, recall that a local ring $A$ is called Cohen-Macaulay if there exists a regular sequence $a_{1}, \ldots, a_{n}(n=\operatorname{dim}(A))$, belonging to the maximal ideal; $a_{1}$ is a non-zero divisor in $A, a_{2}$ is a non-zero divisor in $A /\left\langle a_{1}\right\rangle_{A}$, and so on ([21], Ch. 6). In general the length of a regular sequence does not exceed the dimension of the ring. Therefore a Cohen-Macaulay ring possesses the possibly longest regular sequence, and it is known by this property the intersection theory, or the theory of multiplicity, works very well ([7], Prop. 7.1). A regular local ring, for example $\mathcal{O}_{n}$, is Cohen-Macaulay. In fact, in $\mathcal{O}_{n}$, the coordinate functions $x_{1}, \ldots, x_{n}$ form a regular sequence. If $\mathcal{O}_{n} / \mathcal{J}$ is Cohen-Macaulay for an ideal $\mathcal{J} \subset \mathcal{O}_{n}$, then we call the zero locus $Z(\mathcal{J})$ of $\mathcal{J}$ with the defining ideal $\mathcal{J}$ 
Cohen-Macaulay variety in $\mathbf{C}^{n}$. Non-singular submanifolds are surely Cohen-Macaulay varieties. Another class of important examples for Cohen-Macaulay varieties is given by "determinantal varieties". In fact we have used the following fundamental algebraic fact:

Proposition 1.5 ([13], see also [4]). Let $A$ be a Cohen-Macaulay local ring. Then, for the ideal $\mathcal{J}$ generated by $\ell$-minors of an $n \times m$-matrix with entries in $A$, the quotient ring $A / \mathcal{J}$ is Cohen-Macaulay, provided $\operatorname{dim}(A)-\operatorname{dim}(A / \mathcal{J})=(n+1-\ell)(m+1-\ell)$, the "ideal" codimension.

We have applied Proposition 1.5 in the above proof of Theorem 1.2 to $A=\mathcal{O}_{C}$, $n=r+2, m=r+3$ and $\ell=r+2$. We can also apply it to show Theorem 1.1.

The usage of the Cohen-Macaulay property for the problem of counting perturbed isolated singularities is first considered in [3], along the general framework of intersection theory. Later the original idea is re-explained in [8] for obtaining the new result on the number of double folds. Note that the idea itself of counting perturbed singularities is considered through the deep investigations of germs $\mathbf{C}^{2} \rightarrow \mathbf{C}^{3}$ due to Mond (cf. [22], [23], [24]). See also [30], [28], [29], [19], [20] for the related studies. After [3], [8], there appears a sequence of investigations on the problem of counting general isolated ThomBoardman singularities [26], [4], [5], [6]. Then we observe that there are two main points for obtaining right formulae: one is seeking the appropriate defining ideals of treating singularities on jet spaces and second is seeing their Cohen-Macaulay property. For complicated singularities, both points cause non-trivial problems. In the following sections we collect, around the problems of caustics and wavefronts, several situations where we can overcome these points.

The singularities of generating families of functions can be studied from the viewpoint of singularities of mappings: Let us consider again a Morse family $F:\left(\mathbf{C}^{r} \times \mathbf{C}^{2}, 0\right) \rightarrow \mathbf{C}$ for plane caustic. Then we consider $G:=(F, \Pi):\left(\mathbf{C}^{r} \times \mathbf{C}^{2}, 0\right) \rightarrow \mathbf{C} \times \mathbf{C}^{2}, G(x, \lambda)=$ $(F(x, \lambda), \lambda)$. Then the critical locus of $G$ coincides with the catastrophe set $C(F)$ of $F$. Moreover the cusp (namely, $\Sigma^{r, 1,0}$ ) singular points of $G$ are exactly the fold (namely, $\Sigma^{1,0}$ ) singular points of $\left.\Pi\right|_{C(F)}$, while the swallowtail (namely, $\Sigma^{r, 1,1,0}$ ) singular points of $G$ are exactly the cusp (namely, $\Sigma^{1,1,0}$ ) singular points of $\left.\Pi\right|_{C(F)}$. The $\mathcal{R}^{+}$-stable deformation of $F$ induces a stable deformation of $G$. Therefore Theorem 1.2 follows, for instance, from the following (Corollary 4.4(3) of [4]):

TheOREm 1.6 (Fukui, Nuño Ballesteros, Saia). The number of $\Sigma^{n-2,1,1,0}$-points for a stable perturbation of a $\mathcal{K}$-finite map-germ $G:\left(\mathbf{C}^{n}, 0\right) \rightarrow\left(\mathbf{C}^{3}, 0\right)$ with corank 1 is equal to $\operatorname{dim}_{\mathbf{C}} \mathcal{O}_{n} / \mathcal{J}_{n-2,1,1}(G)$, where $\mathcal{J}_{n-2,1,1}(G)$ is generated by 3 -minors $M_{1}, \ldots$, of Jacobi matrix $\operatorname{Jac}(G)$ of $G,(n-2)$-minors $N_{1}, \ldots$, of $\operatorname{Jac}(G, M)$ and $(n-2)$-minors $L_{1}, \ldots$, of $\operatorname{Jac}(G, M, N)$.

Note that, if $G=(F, \Pi)$, the ideal $\mathcal{J}_{n-2,1,1}(G)$ defined in the above theorem coincides with the ideal treated in Theorem 1.2. Thus we have the second proof of Theorem 1.2.

The problem of counting the number of cusps of caustics is closely related to the problem of counting the number of swallowtails of wave fronts. In fact, $\left.G\right|_{C(F)}: C(F) \rightarrow$ $\mathbf{C} \times \mathbf{C}^{2}$ is the wave front of the Legendrian lifting $\widetilde{L}(F): C(F) \rightarrow \mathbf{C} \times T^{*} \mathbf{C}^{2}$ of the Lagrangian immersion $L(F): C(F) \rightarrow T^{*} \mathbf{C}^{2}$. In fact $\left.F\right|_{C(F)}: C(F) \rightarrow \mathbf{C}$ is a generating 
function of $L(F)$ since $d F=\frac{\partial F}{\partial \lambda_{1}} d \lambda_{1}+\frac{\partial F}{\partial \lambda_{2}} d \lambda_{2}$ on $C(F)=\left\{\frac{\partial F}{\partial x_{1}}=0, \ldots, \frac{\partial F}{\partial x_{r}}=0\right\}$. Thus actually the formula of Theorem 1.2 can be interpreted, from the singularity theory of mappings, as the formula for the number of swallowtails of perturbed wave front. Of course, since swallowtails project to cusps, we have the same formula.

In Legendrian singularity theory, the singularities of wave fronts are analysed via generating families of hypersurfaces [1]. So we give the counting formula for swallowtails of wave fronts in the language of generating families of hypersurfaces.

Let $H:\left(\mathbf{C}^{r} \times \mathbf{C}^{3}, 0\right) \rightarrow(\mathbf{C}, 0)$ be a function family. Assume the equation $H=0$ defines a Morse family of hypersurfaces in $\mathbf{C}^{r}$. This means that the big singular set

$$
\widetilde{C}(H):=\left\{(x, \lambda) \mid H=0, \frac{\partial H}{\partial x_{1}}=0, \ldots, \frac{\partial H}{\partial x_{r}}=0\right\}
$$

is non-singular. The image $\Pi(\widetilde{C}(H))$ of $\widetilde{C}(H)$ under the projection $\Pi: \mathbf{C}^{r} \times \mathbf{C}^{3} \rightarrow \mathbf{C}^{3}$ is called the discriminant of $H$. This coincides with the front of the Legendrian immersion defined by $H$. In fact the mapping $\widetilde{L}(H): \widetilde{C}(H) \rightarrow P T^{*} \mathbf{C}^{3}$ defined by

$$
\widetilde{L}(H)(x, \lambda)=\left(\left[\frac{\partial H}{\partial \lambda_{1}}, \frac{\partial H}{\partial \lambda_{2}}, \frac{\partial H}{\partial \lambda_{3}}\right], \lambda_{1}, \lambda_{2}, \lambda_{3}\right)
$$

is a Legendre immersion in the projective cotangent bundle $P T^{*} \mathbf{C}^{3}$ over $\mathbf{C}^{3}$. For the Legendre fibration $\pi: P T^{*} \mathbf{C}^{3} \rightarrow \mathbf{C}^{3}$, the front of $\widetilde{L}(H)$, that is the image of $\pi \circ \widetilde{L}(H)$, coincides with the discriminant $\Pi(\widetilde{C}(H))$. Any Legendre immersion to $P T^{*} \mathbf{C}^{3}$ is obtained by this process, up to parametrisation.

THEOREM 1.7. The number of swallowtails in the fronts of a stable perturbation of $\widetilde{L}(H)$ is equal to the dimension $\operatorname{dim}_{\mathbf{C}} \widetilde{Q}$ of the algebra

$$
\widetilde{Q}:=\mathcal{O}_{r+3} /\left\langle H, \frac{\partial H}{\partial x_{1}}, \ldots, \frac{\partial H}{\partial x_{r}}, J(\nabla H), J_{1}^{(2)}(\nabla H), \ldots, J_{r}^{(2)}(\nabla H)\right\rangle_{\mathcal{O}_{r+3}},
$$

provided $\operatorname{dim}_{\mathbf{C}} \widetilde{Q}<\infty$.

Proof. Up to $\mathcal{K}$-equivalence, we may assume that $H=F-\lambda_{3}$ for a Morse family $F\left(x, \lambda_{1}, \lambda_{2}\right)$. Then we apply Theorem 1.6 to $G:\left(\mathbf{C}^{r} \times \mathbf{C}^{2}, 0\right) \rightarrow \mathbf{C} \times \mathbf{C}^{2}$, where $G\left(x, \lambda_{1}, \lambda_{2}\right)=\left(F(x, \lambda), \lambda_{1}, \lambda_{2}\right)$.

Note that a more geometric formula is known ([27], [18], [11], [12]) on the number of cusps appearing in a generic plane section of the discriminant by means of Milnor number. However it seems to be open yet to relate it to the formula in term of the iterated Jacobians.

In the following sections we consider several generalisations of the above results including singular cases and try to understand them clearly: In Section 2, we introduce the counting formulae for the numbers of $A_{4}$ and $D_{4}$ singularities of caustics due to Fukui and Weyman. In Section 3, we count the numbers of simplest singularities of singular Lagrangian immersions: open Whitney umbrellas. In Section 4, we count isolated singularities of singular Lagrangian immersions composed with Lagrangian projections. Actually, as the result obtained firstly in this paper, we give the formulae for the numbers of singularities $S_{3}, S_{4}, S_{5}$ and $T_{5}$ introduced in [2]. 
In the last section, we give open questions. We do not treat in this paper the interesting problem on the algebraic equations for the number of real cusps and other isolated singularities over the real. Also we do not treat the topological invariance of the complex or real numbers of isolated singularities and Euler characteristics of non-isolated singularities of stable perturbations.

The present paper is based partly on my talk under the same title at Yokohama, Japan, on November 2000, in the occasion of the celebration for the 60th birthdays of Professor Fukuda and of Professor Izumi. I would like to thank Professor Toshizumi Fukui and Professor Jerzy Weyman for their encouragement to my writing of this note by their asking the question on perturbations of caustics. I would like to thank Professor D. Siersma for his reminding me the other geometric formulae on the numbers of cusps. I would like to thank organisers of the conference Caustics'02, for their encouragement to my completing of the present paper.

2. Space caustics. So far we have investigated the number of $A_{3}$ singularities of caustics on the plane. Then it is natural to ask about the number of $A_{4}$ and $D_{4}$ singularities of caustics in the three space [1].

REMARK 2.1. The number of $A_{2}$-singularities of a generating family $F:\left(\mathbf{C}^{r} \times\right.$ $\mathbf{C}, 0) \rightarrow \mathbf{C}, F=F(x, \lambda)$ is given by $\operatorname{dim}_{\mathbf{C}} \mathcal{O}_{r+1} / \mathcal{J}_{r, 1}, \mathcal{J}_{r, 1}=\langle\nabla F, J(\nabla F)\rangle$.

Let $F:\left(\mathbf{C}^{r} \times \mathbf{C}^{3}, 0\right) \rightarrow \mathbf{C}$ be a generating Morse family of a Lagrangian immersion $f:\left(\mathbf{C}^{3}, 0\right) \rightarrow T^{*} \mathbf{C}^{3}$. The fold $\left(A_{2}\right)$ locus of $\left.\Pi\right|_{C(F)}$ is defined by $\nabla F=0$ and $J(\nabla F)=0$. The cusp $\left(A_{3}\right)$ locus of $\left.\Pi\right|_{C(F)}$ is defined by $\nabla F, J(\nabla F)$ and $J_{1}^{(2)}(\nabla F), \ldots, J_{r}^{(2)}(\nabla F)$. Then, for the description of swallowtail $\left(A_{4}\right)$ locus of $\left.\Pi\right|_{C(F)}$, we intend to consider the mapping

$$
\left(\nabla F, J(\nabla F), J_{1}^{(2)}(\nabla F), \ldots, J_{r}^{(2)}(\nabla F), \lambda_{1}, \lambda_{2}, \lambda_{3}\right):\left(\mathbf{C}^{r+3}, 0\right) \rightarrow\left(\mathbf{C}^{2 r+4}, 0\right),
$$

as in the proof of Theorem 1.2. However actually there is no reason to expect that its singular locus coincides with the swallowtail locus of $\left.\Pi\right|_{C(F)}$, because

$$
\nabla F, J(\nabla F), J_{1}^{(2)}(\nabla F), \ldots, J_{r}^{(2)}(\nabla F)
$$

are far from being independent. In fact, in [4], [5] it is observed that the singular locus includes the locus of $D_{4}$ as well as the locus of $A_{4}$.

Anyway we define the ideal $\mathcal{J}_{r, 1,1,1}$ in $\mathcal{O}_{r+3}$ generated by

$$
\nabla F, J(\nabla F), J_{1}^{(2)}(\nabla F), \ldots, J_{r}^{(2)}(\nabla F)
$$

and Jacobians of $r$ elements from $\nabla F, J(\nabla F), J_{1}^{(2)}(\nabla F), \ldots, J_{r}^{(2)}(\nabla F)$ with respect to $x_{1}, \ldots, x_{r}$. Moreover we define the ideal $\mathcal{J}_{r, 2}$ in $\mathcal{O}_{r+3}$ generated by $\nabla F$ and $(r-1)$ minors of the Jacobi matrix $\operatorname{Jac}(\nabla F)$ of $\nabla F$. Then the following result is the remarkable Theorem 3.2 of [5] rewritten in the language of generating family.

Theorem 2.2 (Fukui-Weyman [5]). Let $F:\left(\mathbf{C}^{r} \times \mathbf{C}^{3}, 0\right) \rightarrow \mathbf{C}$ be a Morse family. Then we have the formulae for the number $\# D_{4}$ of $D_{4}$-singular points and the number $\# A_{4}$ of $A_{4}$-singular points, respectively, after a Lagrange stable perturbation:

$$
\# D_{4}=\operatorname{dim}_{\mathbf{C}} \mathcal{O}_{r+3} / \mathcal{J}_{r, 2},
$$


and

$$
\# A_{4}=\operatorname{dim}_{\mathbf{C}} \mathcal{O}_{r+3} / \mathcal{J}_{r, 1,1,1}-4 \operatorname{dim}_{\mathbf{C}} \mathcal{O}_{r+3} / \mathcal{J}_{r, 2},
$$

provided $\operatorname{dim}_{\mathbf{C}} \mathcal{O}_{r+3} / \mathcal{J}_{r, 2}<\infty$ and $\operatorname{dim}_{\mathbf{C}} \mathcal{O}_{r+3} / \mathcal{J}_{r, 1,1,1}<\infty$.

ExAmple 2.3. Consider the generating family of type $D_{4}$ :

$$
F\left(x_{1}, x_{2}, \lambda_{1}, \lambda_{2}, \lambda_{3}\right)=x_{1}^{2} x_{2}+x_{2}^{3}+\lambda_{1} x_{2}^{2}+\lambda_{2} x_{1}+\lambda_{3} x_{2},
$$

$(r=2)$. Then

$$
\operatorname{dim}_{\mathbf{C}} \mathcal{O}_{5} / \mathcal{J}_{2,2}=\operatorname{dim}_{\mathbf{C}} \mathcal{O}_{5} /\left\langle\frac{\partial F}{\partial x_{1}}, \frac{\partial F}{\partial x_{2}}, \frac{\partial^{2} F}{\partial x_{1}^{2}}, \frac{\partial^{2} F}{\partial x_{1} \partial x_{2}}, \frac{\partial^{2} F}{\partial x_{2}^{2}}\right\rangle_{\mathcal{O}_{5}}=1
$$

Moreover we have

$$
\mathcal{J}_{2,1,1,1}=\left\langle x_{1}^{2}, x_{1} x_{2}, x_{2}^{2}, \lambda_{1} x_{1}, \lambda_{1} x_{2}, \lambda_{1}^{2}, \lambda_{2}, \lambda_{3}\right\rangle_{\mathcal{O}_{5}} .
$$

Thus we see $\mathcal{O}_{5} / \mathcal{J}_{2,1,1,1}$ has a basis $\left\{1, x_{1}, x_{2}, \lambda_{1}\right\}$ and

$$
\operatorname{dim}_{\mathbf{C}} \mathcal{O}_{5} / \mathcal{J}_{2,1,1,1}=4
$$

for $D_{4}$. On the other hand, for the generating family of type $A_{4}$ :

$$
F\left(x_{1}, x_{2}, \lambda_{1}, \lambda_{2}, \lambda_{3}\right)=x_{1}^{5}+x_{2}^{2}+\lambda_{1} x_{1}^{3}+\lambda_{2} x_{1}^{2}+\lambda_{3} x_{1},
$$

$(r=2)$, we have $\operatorname{dim}_{\mathbf{C}} \mathcal{O}_{5} / \mathcal{J}_{2,2}=0$ and $\mathcal{O}_{5} / \mathcal{J}_{2,1,1,1}=1$.

Let $f:\left(\mathbf{C}^{3}, 0\right) \rightarrow T^{*} \mathbf{C}^{3}$ be a Lagrangian immersion and $\pi: T^{*} \mathbf{C}^{3} \rightarrow \mathbf{C}^{3}$ the canonical projection. We can re-formulate Theorem 2.2, without using the term of generating family, as follows: Considering the composition $\pi \circ f=\left(q_{1} \circ f, q_{2} \circ f, q_{3} \circ f\right)$, we define $\mathcal{J}_{2}$ as the ideal generated by the 2-minors of the Jacobi matrix $\operatorname{Jac}\left(q_{1} \circ f, q_{2} \circ f, q_{3} \circ f\right)$ and define $\mathcal{J}_{1,1,1}$ as the ideal generated by the Jacobian $J=J\left(q_{1} \circ f, q_{2} \circ f, q_{3} \circ f\right)$, the secondary Jacobians

$$
J_{1}^{(2)}=\left(J, q_{2} \circ f, q_{3} \circ f\right), J_{2}^{(2)}=J\left(q_{1} \circ f, J, q_{3} \circ f\right), J_{3}^{(2)}=J\left(q_{1} \circ f, q_{2} \circ f, J\right)
$$

and the trinary Jacobians, namely, 3-minors of

$$
\operatorname{Jac}\left(q_{1} \circ f, q_{2} \circ f, q_{3} \circ f, J, J_{1}^{(2)}, J_{2}^{(2)}, J_{3}^{(2)}\right) .
$$

Then we have:

Corollary 2.4. Let $f:\left(\mathbf{C}^{3}, 0\right) \rightarrow T^{*} \mathbf{C}^{3}$ be a Lagrangian immersion. Then the number $\# D_{4}$ of $D_{4}$-singularities and \# $A_{4}$ of $A_{4}$-singularities respectively appearing in a Lagrange stable perturbation of $f$ is given by

$$
\# D_{4}=\operatorname{dim}_{\mathbf{C}} \mathcal{O}_{3} / \mathcal{J}_{2}, \quad \# A_{4}=\operatorname{dim}_{\mathbf{C}} \mathcal{O}_{3} / \mathcal{J}_{1,1,1}-4 \operatorname{dim}_{\mathbf{C}} \mathcal{O}_{3} / \mathcal{J}_{2},
$$

provided $\operatorname{dim}_{\mathbf{C}} \mathcal{O}_{3} / \mathcal{J}_{2}<\infty$ and $\operatorname{dim}_{\mathbf{C}} \mathcal{O}_{3} / \mathcal{J}_{1,1,1}<\infty$.

Proof. Let $F:\left(\mathbf{C}^{r} \times \mathbf{C}^{3}, 0\right) \rightarrow \mathbf{C}$ be a generating family of $f$. We may suppose $\varphi:=\left.\pi_{r}\right|_{C(F)}:(C(F), 0) \rightarrow\left(\mathbf{C}^{r}, 0\right), C(F)$ being the catastrophe set-germ, is a complex analytic isomorphism. Also consider the inclusion $i:(C(F), 0) \rightarrow\left(\mathbf{C}^{r} \times \mathbf{C}^{3}, 0\right)$. Then the composition of $i^{*}: \mathcal{O}_{r+3} \rightarrow \mathcal{O}_{C(F)}$ with the inverse of $\varphi^{*}: \mathcal{O}_{r} \rightarrow \mathcal{O}_{C(F)}$ induces the isomorphisms

$$
\mathcal{O}_{r+3} / \mathcal{J}_{r, 2} \cong \mathcal{O}_{3} / \mathcal{J}_{2}, \quad \text { and } \quad \mathcal{O}_{r+3} / \mathcal{J}_{r, 1,1,1} \cong \mathcal{O}_{3} / \mathcal{J}_{1,1,1}
$$


Here we also give the result on the numbers of $A_{4}$ and $D_{4}$ for generating families $H\left(x_{1}, \ldots, x_{r}, \lambda_{0}, \lambda_{1}, \ldots, \lambda_{3}\right)=0$ on $\left(\mathbf{C}^{r} \times \mathbf{C}^{4}, 0\right)$ of Legendre immersions $\left(\mathbf{C}^{3}, 0\right) \rightarrow$ $P T^{*} \mathbf{C}^{4}$. We define, for $H$, similarly as above, ideals $\widetilde{\mathcal{J}}_{r, 2}$ and $\widetilde{\mathcal{J}}_{r, 1,1,1}$ in $\mathcal{O}_{r+4}$, but adding $H$ to the generators of $\mathcal{J}_{r, 2}$ and $\mathcal{J}_{r, 1,1,1}$ respectively. Then we have

Corollary 2.5. Let $H:\left(\mathbf{C}^{r} \times \mathbf{C}^{4}, 0\right) \rightarrow(\mathbf{C}, 0)$ be a Morse generating family of hypersurfaces for a Legendrian immersion $\widetilde{L}(H): \widetilde{C}(H) \rightarrow P T^{*} \mathbf{C}^{4}$. Then the numbers $\# D_{4}$ and $\# A_{4}$ of $D_{4}$ and $A_{4}$ singularities respectively of a Legendrian perturbation of $\widetilde{L}(H)$ are given by:

$$
\# D_{4}=\operatorname{dim}_{\mathbf{C}} \mathcal{O}_{r+4} / \tilde{\mathcal{J}}_{r, 2}
$$

and

$$
\# A_{4}=\operatorname{dim}_{\mathbf{C}} \mathcal{O}_{r+4} / \tilde{\mathcal{J}}_{r, 1,1,1}-4 \operatorname{dim}_{\mathbf{C}} \mathcal{O}_{r+4} / \tilde{\mathcal{J}}_{r, 2}
$$

provided $\operatorname{dim}_{\mathbf{C}} \mathcal{O}_{r+4} / \widetilde{\mathcal{J}}_{r, 2}<\infty$ and $\operatorname{dim}_{\mathbf{C}} \mathcal{O}_{r+4} / \widetilde{\mathcal{J}}_{r, 1,1,1}<\infty$.

REMARK 2.6. The counting of isolated singularities of caustics in the four space has several difficulties: The ideals associated to Thom-Boardman singularities are actually defined in general ([25], [26], [4], [5]): They are called Morin ideals of Thom-Boardman singularities. However, for $A_{5}$-singularities or $\Sigma^{r, 1,1,1,1}$-singularities, the Morin ideal does not define a Cohen-Macaulay variety (Theorem 3.1 of [5]). Moreover we do not know the coefficients of necessary correcting terms for the counting of the number of $\Sigma^{r, 1,1,1,1}$ singularities. Furthermore, we note that $D_{5}$-singularity of caustics is no longer a ThomBoardman singularity. So we need, in general, a method to find the defining ideals for closures of $\mathcal{K}$-orbits in jet spaces.

3. Open Whitney umbrellas. Let $f:\left(\mathbf{C}^{n}, 0\right) \rightarrow T^{*} \mathbf{C}^{n}$ be an isotropic mapping; $f^{*} \omega=0$. In this and in the next sections, we do not assume $f$ is an immersion (i.e. of corank 0). Instead we do assume $f$ is of corank not exceeding 1 .

In this section we do not treat singularities of caustics nor singularities of Lagrangian projections. Instead we do treat the singularities of $f$ up to the symplectic equivalence [16]. Actually we consider Thom-Boardman singularities of type $\Sigma^{1,1, \ldots}$ for isotropic mappings.

In this paper, by the isotropic jet space we mean the space of isotropic jets of corank not exceeding 1:

$$
J_{I}^{r}\left(\mathbf{C}^{n}, T^{*} \mathbf{C}^{n}\right):=\left\{j^{r} f(x) \mid x \in \mathbf{C}^{n}, f:\left(\mathbf{C}^{n}, x\right) \rightarrow T^{*} \mathbf{C}^{n}, f^{*} \omega=0, \operatorname{corank}(f) \leq 1\right\} .
$$

Moreover we set

$$
J_{I}^{r}(n, 2 n):=\left\{j^{r} f(0) \mid f:\left(\mathbf{C}^{n}, 0\right) \rightarrow\left(T^{*} \mathbf{C}^{n}, 0\right), f^{*} \omega=0, \operatorname{corank}(f) \leq 1\right\} .
$$

Recall that the Thom-Boardman singularity $\Sigma^{1_{k}}=\Sigma^{1,1, \ldots}$ ( $k$ times) is defined in the ordinary jet space $J^{r}\left(\mathbf{C}^{n}, T^{*} \mathbf{C}^{n}\right)(k \leq r) ; \Sigma^{1_{k}}\left(\mathbf{C}^{n}, T^{*} \mathbf{C}^{n}\right) \subseteq J^{r}\left(\mathbf{C}^{n}, T^{*} \mathbf{C}^{n}\right)$ is a submanifold of codimension $(n+1) k$. Then simply we set

$$
\Sigma_{I}^{1_{k}}\left(\mathbf{C}^{n}, T^{*} \mathbf{C}^{n}\right):=\Sigma^{1_{k}}\left(\mathbf{C}^{n}, T^{*} \mathbf{C}^{n}\right) \cap J_{I}^{r}\left(\mathbf{C}^{n}, T^{*} \mathbf{C}^{n}\right) .
$$

Similarly, for $\Sigma^{1_{k}}(n, 2 n) \subseteq J^{r}(n, 2 n)$, we set

$$
\Sigma_{I}^{1_{k}}(n, 2 n):=\Sigma^{1_{k}}(n, 2 n) \cap J_{I}^{r}(n, 2 n) .
$$


Then, via the trivialisation

$$
J^{r}\left(\mathbf{C}^{n}, T^{*} \mathbf{C}^{n}\right) \cong \mathbf{C}^{n} \times T^{*} \mathbf{C}^{n} \times J^{r}(n, 2 n),
$$

we have

$$
J_{I}^{r}\left(\mathbf{C}^{n}, T^{*} \mathbf{C}^{n}\right) \cong \mathbf{C}^{n} \times T^{*} \mathbf{C}^{n} \times J_{I}^{r}(n, 2 n)
$$

and

$$
\Sigma_{I}^{1_{k}}\left(\mathbf{C}^{n}, T^{*} \mathbf{C}^{n}\right) \cong \mathbf{C}^{n} \times T^{*} \mathbf{C}^{n} \times \Sigma_{I}^{1_{k}}(n, 2 n) .
$$

We abbreviate $J^{r}\left(\mathbf{C}^{n}, T^{*} \mathbf{C}^{n}\right)$ by $J^{r}$, and similarly we abbreviate $J_{I}^{r}\left(\mathbf{C}^{n}, T^{*} \mathbf{C}^{n}\right)$ (resp. $\left.\Sigma_{I}^{1_{k}}\left(\mathbf{C}^{n}, T^{*} \mathbf{C}^{n}\right)\right)$ by $J_{I}^{r}\left(\operatorname{resp} . \Sigma_{I}^{1_{k}}\right)$.

It is known that the isotropic map-germ of corank not greater than 1 is essentially described by $J^{r}(1,2)$ up to symplectic equivalence ([14], [15], [16], [17]). In particular we have:

LEMMA 3.1 ([14]). $\Sigma_{I}^{1_{k}}(n, 2 n)$ is a submanifold of $J_{I}^{r}(n, 2 n)$ of codimension $2 k$. $\Sigma_{I}^{1_{k}}=\Sigma_{I}^{1_{k}}\left(\mathbf{C}^{n}, T^{*} \mathbf{C}^{n}\right)$ is a submanifold of $J_{I}^{r}=J_{I}^{r}\left(\mathbf{C}^{n}, T^{*} \mathbf{C}^{n}\right)$ of codimension $2 k$.

Generic isotropic mappings of corank not greater than 1 are classified into open Whitney umbrellas of type $k(0 \leq k \leq[n / 2])([14])$. They are characterised by the symplectic stability [16]. Among them, the classification coincides with the classification by ThomBoardman singularities: An open Whitney umbrella $f:\left(\mathbf{C}^{n}, x\right) \rightarrow\left(T^{*} \mathbf{C}^{n}, f(x)\right)$ is of type $k$ if and only if $j^{r} f(x) \in \Sigma_{I}^{1_{k}, 0}\left(=\Sigma_{I}^{1_{k}} \backslash \Sigma_{I}^{1_{k+1}}\right)$. We abbreviate an open Whitney umbrella of type $k$ by $\mathrm{OWU}_{k}$.

For an isotropic mapping $f:\left(\mathbf{C}^{2 n}, 0\right) \rightarrow T^{*} \mathbf{C}^{2 n}$, open Whitney umbrellas of type $n$ appear isolatedly by Lemma 3.1 after a symplectically stable perturbation. Then we naturally have the question:

How many open Whitney umbrellas of isolated type do there appear after a symplectically stable perturbation?

We define, in general, for a map-germ $f:\left(\mathbf{C}^{m}, 0\right) \rightarrow\left(\mathbf{C}^{p}, 0\right)$ with $m \leq p$, the ideal $\mathcal{J}_{1_{k}}$ of $\mathcal{O}_{m}$ generated by $m$-minors $M_{1}^{(1)}, M_{2}^{(1)}, \ldots$, of the Jacobi matrix (an $m \times p$-matrix) $\operatorname{Jac}(f)$ of $f, m$-minors $M_{1}^{(2)}, M_{2}^{(2)}, \ldots$, of the Jacobi matrix (an $m \times\left(p+\left(\begin{array}{c}p \\ m\end{array}\right)\right)$-matrix) $\operatorname{Jac}\left(f, M^{(1)}\right), m$-minors $M_{1}^{(3)}, M_{2}^{(3)}, \ldots$, of $\operatorname{Jac}\left(f, M^{(1)}, M^{(2)}\right), \ldots$, and lastly $m$-minors of $\operatorname{Jac}\left(f, M^{(1)}, M^{(2)}, \ldots, M^{(k-1)}\right)$.

Then we have:

Proposition 3.2. Let $f:\left(\mathbf{C}^{2 n}, 0\right) \rightarrow T^{*} \mathbf{C}^{2 n}$ be an isotropic map-germ of corank not exceeding 1 . Then the number $\# \mathrm{OWU}_{n}$ of open Whitney umbrellas of type $n$ after a symplectically stable perturbation of $f$ is given by

$$
\# \mathrm{OWU}_{n}=\operatorname{dim}_{\mathbf{C}} \mathcal{O}_{2 n} / \mathcal{J}_{1_{n}}
$$

provided $\operatorname{dim}_{\mathbf{C}} \mathcal{O}_{2 n} / \mathcal{J}_{1_{n}}<\infty$.

Proof. The corresponding ideal to $\mathcal{J}_{1_{n}}$ in $\mathcal{O}_{J^{n}}$ is a regular defining ideal of $\Sigma^{1_{n}}$. Moreover it gives a regular defining ideal in $\mathcal{O}_{J_{I}^{n}}$ of $\Sigma_{I}^{1_{n}}$, which is non-singular of codimension $2 n$. This means that, at each point of $\Sigma_{I}^{1_{n}}$, there are $2 n$ elements from the ideal which give a submersion at the point. The number of open Whitney umbrellas of type $n$ 
after a symplectically stable perturbation is equal to the intersection number of $J^{n} f\left(\mathbf{C}^{2 n}\right)$ and $\Sigma_{I}^{1_{n}}$ in $J_{I}^{n}=J_{I}^{n}\left(\mathbf{C}^{2 n}, T^{*} \mathbf{C}^{2 n}\right)$. This implies the result.

In particular, setting $n=1$ in Proposition 3.2, we have the following result.

Corollary 3.3. Let $f:\left(\mathbf{C}^{2}, 0\right) \rightarrow T^{*} \mathbf{C}^{2}$ be an isotropic map-germ of corank not greater than 1. Denote by $u$ the number of open Whitney umbrellas of symplectically stable perturbations of $f$. Then

$$
u=\operatorname{dim}_{\mathbf{C}} \mathcal{O}_{2} / \mathcal{J}_{1}
$$

where $\mathcal{J}_{1}$ is the ideal generated by 2-minors of Jacobi matrix $\operatorname{Jac}(f)$ of $f$.

We apply the above result to the problem of counting the number of folded umbrellas of fronts.

A map-germ $g:\left(\mathbf{C}^{n}, 0\right) \rightarrow\left(\mathbf{C}^{n+1}, 0\right)$ with dense immersion locus is called a frontal map-germ if, for any $t \in\left(\mathbf{C}^{n}, 0\right)$, there exists unique limit of tangent spaces through immersive points $\lim _{s \rightarrow t} g_{*}\left(T_{s} \mathbf{C}^{n}\right)=: T_{t}$ depending smoothly on $t$.

For example, the swallowtail $\left(x_{1}, x_{2}\right) \mapsto\left(x_{1}, x_{2}^{3}+x_{1} x_{2}, \frac{3}{4} x_{2}^{4}+\frac{1}{2} x_{1} x_{2}^{2}\right)$ and the folded umbrella $\left(x_{1}, x_{2}\right) \mapsto\left(x_{1}, x_{2}^{2}, x_{1} x_{2}^{3}\right)$ are frontal, while the Whitney umbrella $\left(x_{1}, x_{2}\right) \mapsto$ $\left(x_{1}, x_{2}^{2}, x_{1} x_{2}\right)$ is not frontal, when they are regarded as map-germs $\left(\mathbf{C}^{2}, 0\right) \rightarrow\left(\mathbf{C}^{3}, 0\right)$.

Let $g:\left(\mathbf{C}^{n}, 0\right) \rightarrow\left(\mathbf{C}^{n+1}, 0\right)$ be frontal. Then the map-germ to the Grassmannian

$$
G:\left(\mathbf{C}^{n}, 0\right) \rightarrow \operatorname{Gr}\left(n, T \mathbf{C}^{n+1}\right)=P T^{*} \mathbf{C}^{n+1}
$$

defined by $G(t)=T_{t}$ is an integral map-germ: If we consider the contact form $\alpha=d r-\sum_{i=1}^{n} p_{i} d q_{i}$ on $\mathbf{C}^{2 n+1}=J^{1}\left(\mathbf{C}^{n}, \mathbf{C}\right) \subset P T^{*} \mathbf{C}^{n+1}$, then $G^{*} \alpha=0$ provided $G(0) \in J^{1}\left(\mathbf{C}^{n}, \mathbf{C}\right)$. For integral mappings see [1]. Then we have the following:

Proposition 3.4. Let $g:\left(\mathbf{C}^{2 n}, 0\right) \rightarrow\left(\mathbf{C}^{2 n+1}, 0\right)$ be a frontal mapping with the integral lifting $G:\left(\mathbf{C}^{2 n}, 0\right) \rightarrow J^{1}\left(\mathbf{C}^{2 n}, \mathbf{C}\right)=\mathbf{C}^{4 n+1}$ of corank not greater than 1 . Set

$$
G=\left(g_{1}, \ldots, g_{2 n}, e ; a_{1}, \ldots, a_{2 n}\right)
$$

with de $=a_{1} d f_{1}+\ldots+a_{2 n} d f_{2 n}$. Then the number \#FU $\mathrm{F}_{n}$ of folded umbrellas of type $n$ after a frontal stable perturbation of $g$ is equal to $\# \mathrm{OWU}_{n}$ for the isotropic map-germ $f=\left(g_{1}, \ldots, g_{2 n}, a_{1}, \ldots, a_{2 n}\right):\left(\mathbf{C}^{2 n}, 0\right) \rightarrow T^{*} \mathbf{C}^{2 n}$. Therefore

$$
\# \mathrm{FU}_{n}=\operatorname{dim}_{\mathbf{C}} \mathcal{O}_{2 n} / \mathcal{J}_{1_{n}}(f) \text {. }
$$

In particular consider the case $n=2$. It is known that any integral map $G:\left(\mathbf{C}^{2}, 0\right) \rightarrow$ $\operatorname{Gr}\left(2, T \mathbf{C}^{2}\right)$ of corank not exceeding 1 is approximated by an integral map $\widetilde{G}$ such that $\widetilde{g}=\pi \circ \widetilde{G}$ has only swallowtails and folded umbrellas as singularities (cf. [9]). Denote by $s$ (resp. $u$ ) the number of swallowtails (resp. folded umbrellas) for perturbations $\widetilde{g}=\pi \circ \widetilde{G}$ from $g$. Then we have:

Corollary 3.5. Let $g:\left(\mathbf{C}^{2}, 0\right) \rightarrow\left(\mathbf{C}^{3}, 0\right)$ be a frontal map-germ with the integral lifting $G:\left(\mathbf{C}^{2}, 0\right) \rightarrow J^{1}\left(\mathbf{C}^{2}, \mathbf{C}\right)=\mathbf{C}^{5}$ of corank not greater than 1 . Set $G=$ $\left(g_{1}, g_{2}, e ; a_{1}, a_{2}\right)$ with de $=a_{1} d g_{1}+a_{2} d g_{2}$. Then we have $s=\operatorname{dim}_{\mathbf{C}} Q$ with

$$
Q:=\mathcal{O}_{2} /\left\langle J\left(g_{1}, g_{2}\right), J\left(g_{1}, J\left(g_{1}, g_{2}\right)\right), J\left(J\left(g_{1}, g_{2}\right), g_{2}\right)\right\rangle_{\mathcal{O}_{2}} .
$$


Moreover we have $u=\operatorname{dim}_{\mathbf{C}} Q^{\prime}$ with

$$
Q^{\prime}:=\mathcal{O}_{2} /\left\langle\begin{array}{l}
J\left(g_{1}, g_{2}\right), J\left(g_{1}, a_{1}\right), J\left(g_{1}, a_{2}\right) \\
J\left(g_{2}, a_{1}\right), J\left(g_{2}, a_{2}\right), J\left(a_{1}, a_{2}\right)
\end{array}\right\rangle_{\mathcal{O}_{2}} .
$$

REMARK 3.6. Lowering more the dimension, consider a plane curve $g:(\mathbf{C}, 0) \rightarrow$ $\left(\mathbf{C}^{2}, 0\right)$ as front. Then the number $\kappa$ of cusps of frontal perturbations of $g$ is equal to the order of the plane curve-germ $g: \kappa=\operatorname{ord}(g)$.

4. Supersingularities of caustics. Here we count isolated singularities of isotropic mappings relatively to the Lagrangian projections, namely singularities of composed isotropic mapping $f:\left(\mathbf{C}^{n}, 0\right) \rightarrow T^{*} \mathbf{C}^{n}$ with the Lagrangian fibration $\pi: T^{*} \mathbf{C}^{n} \rightarrow \mathbf{C}^{n}$. If $f$ is an immersion, then $f$ is a Lagrangian immersion, and the composed mapping $\pi \circ f$ is exactly the Lagrangian mapping of $f$. In general, the singularity of composed mapping is not necessarily reflected to the singularity of the caustic; the set of critical values of the composed mapping $\pi \circ f$. This is the reason to use the term "supersingularity".

We denote by $\pi: T^{*} \mathbf{C}^{n} \rightarrow \mathbf{C}^{n}$ the canonical projection. We define Thom-Boardman singularity $\Sigma_{i_{1}, i_{2}, \ldots, I}^{1_{k}}$ for isotropic mappings of corank not greater than 1 by

$$
\Sigma_{I}^{1_{k}} \cap \pi_{r}^{-1}\left(\Sigma^{i_{1}, i_{2}, \cdots}\right) \subset J_{I}^{r}=J_{I}^{r}\left(\mathbf{C}^{n}, T^{*} \mathbf{C}^{n}\right) .
$$

Here we use the letter " $I$ " for "isotropic", and denote by

$$
\pi_{r}: J_{I}^{r}\left(\mathbf{C}^{n}, T^{*} \mathbf{C}^{n}\right) \rightarrow J^{r}\left(\mathbf{C}^{n}, \mathbf{C}^{n}\right)
$$

the projection induced by $\pi: T^{*} \mathbf{C}^{n} \rightarrow \mathbf{C}^{n}$. Note that the closure $\overline{\Sigma_{i_{1}, i_{2}, \ldots, I}^{1_{k}}}$ in $J_{I}^{r}=$ $J_{I}^{r}\left(\mathbf{C}^{n}, T^{*} \mathbf{C}^{n}\right)$ is equal to

$$
\Sigma_{I}^{1_{k}} \cap \pi_{r}^{-1}\left(\overline{\sum^{i_{1}, i_{2}, \ldots}}\right) .
$$

In [2], we classify simple Lagrangian projections of open Whitney umbrellas of type 1. Moreover we know the "nice range" for isotropic mappings $\mathbf{C}^{n} \rightarrow T^{*} \mathbf{C}^{n}$ of corank not exceeding 1 under the Lagrangian equivalence is $\{n \in \mathbf{N} \mid n \leq 4\}$. This means that any isotropic map-germ $f:\left(\mathbf{C}^{n}, 0\right) \rightarrow T^{*} \mathbf{C}^{n}$ of corank not greater than 1 is perturbed into an isotropic mapping with only Lagrange stable germs at any point in a sufficiently small neighbourhood of 0 , provided $n \leq 4$. Note that a generic isotropic map-germ $\left(\mathbf{C}^{4}, 0\right) \rightarrow T^{*} \mathbf{C}^{4}$ of type $\Sigma^{1,1}$ has unique Lagrange equivalence class (Theorem 3 of [14]). Also note that the nice range for Lagrangian immersions is $\{n \in \mathbf{N} \mid n \leq 5\}$ ([1], [17]).

Now we consider the supersingularities of caustics with $n \leq 4$. From the classification in [2], we have:

TheOREM 4.1. Let $f:\left(\mathbf{C}^{n}, 0\right) \rightarrow T^{*} \mathbf{C}^{n}$ be an isotropic map-germs of corank not exceeding 1 with $n=1,2,3$ or 4 . Then $f$ has a Lagrange stable perturbation with singularities only in the following list:

(1) $n=1 . A_{2}=\Sigma_{1,0, I}^{0}$ the fold.

(2) $n=2$. In addition, $A_{3}=\Sigma_{1,1,0, I}^{0}$ the cusp, and $S_{3}=\Sigma_{1,0, I}^{1,0}$ the (original) open Whitney umbrella.

(3) $n=3$. In addition, $A_{4}=\Sigma_{1,1,1,0, I}^{0}$ the swallowtail, $D_{4}=\Sigma_{2,0, I}^{0}$ the umbilic, and $S_{4}=\Sigma_{1,1,0, I}^{1,0}$. 
(4) $n=4$. In addition, $A_{5}=\Sigma_{1,1,1,0, I}^{0}, D_{5}, S_{5}=\Sigma_{1,1,1,0, I}^{1,0}, T_{5}=\Sigma_{2,0, I}^{1,0}$ and $W_{5}=$ $\Sigma_{1,1,0, I}^{1,1,0}$.

REMARK 4.2. Note that, in the case $n=4$, as a generic isotropic mapping, the open Whitney umbrellas of type 2 appear as well as that of type 1 . Thus we must put, in addition to the list from Theorem 1 of [2], the Lagrangian equivalence classes of generic open Whitney umbrellas of type 2 . However generic projections of open Whitney umbrellas of type 2 has unique Lagrangian equivalence class by Theorem 3 of [14]. In this paper we denote the unique class by $W_{5}$. It was denoted in [14] by $f_{4,2}$. The singularity $T_{5}$, found by Ilya Bogaevskii ([2]) is given by a stable projection of corank two at the singular point of the open Whitney umbrella of type 1 in the smallest dimension.

Now naturally we ask:

What is the algebraic formula for the number of isolated supersingularities after a Lagrange stable perturbation of an isotropic map-germ of corank one?

We give the formulae for the numbers of $A_{2}, A_{3}, S_{3}, S_{4}, S_{5}, W_{5}$ and $T_{5}$ for isotropic map-germs of corank one. For $S_{5}$, yet we have an inequality (Proposition 4.9). We observe naturally a possible similarity between the pair $\left(D_{4}, A_{4}\right)$ and the pair $\left(T_{5}, S_{5}\right)$. Thus we conjecture that the equality for $S_{5}$ in Proposition 4.9 holds.

Now we start with $A_{2}$. The following fact is very easy to see:

Proposition 4.3. Let $f:(\mathbf{C}, 0) \rightarrow T^{*} \mathbf{C}$ be a map-germ. Then the number of $A_{2}$-singular points appearing in a Lagrange stable perturbation of $f$ is given by $\# A_{2}=\operatorname{dim} \mathcal{O}_{1} / \mathcal{J}_{1}$ provided $\operatorname{dim} \mathcal{O}_{1} / \mathcal{J}_{1}<\infty$, where $\mathcal{J}_{1}:=\langle J(\pi \circ f)\rangle$.

Next we turn to $A_{3}$. The formula of $A_{3}$ for a Lagrangian immersion is given in Section 1. Also for isotropic map-germ of corank one, we have the following:

Proposition 4.4. Let $f:\left(\mathbf{C}^{2}, 0\right) \rightarrow T^{*} \mathbf{C}^{2}$ be an isotropic map-germ. Then the number of $A_{3}$-singular points appearing in a Lagrange stable perturbation of $f$ is given by

$$
\# A_{3}=\operatorname{dim} \mathcal{O}_{2} / \mathcal{J}_{1,1}
$$

provided $\operatorname{dim} \mathcal{O}_{2} / \mathcal{J}_{1,1}<\infty$, where we set as before

$$
\mathcal{J}_{1,1}:=\left\langle J\left(f_{1}, f_{2}\right), J\left(f_{1}, J\left(f_{1}, f_{2}\right)\right), J\left(J\left(f_{1}, f_{2}\right), f_{2}\right)\right\rangle,
$$

setting $\pi \circ f=\left(f_{1}, f_{2}\right):\left(\mathbf{C}^{2}, 0\right) \rightarrow\left(\mathbf{C}^{2}, 0\right)$.

Proof. Since a Lagrange stable perturbation of $f$ induces a stable perturbation of $\pi \circ f$, in this case, Proposition 4.4 follows from Theorem 1.1.

Next we proceed to count supersingularities. First we note that $\# S_{3}=\# \mathrm{OWU}_{1}$ (resp. $\# W_{5}=\# \mathrm{OWU}_{2}$ ) for a Lagrange stable perturbation of $f:\left(\mathbf{C}^{2}, 0\right) \rightarrow T^{*} \mathbf{C}^{2}$ (resp. $\left.f:\left(\mathbf{C}^{4}, 0\right) \rightarrow T^{*} \mathbf{C}^{4}\right)$. Thus already we have given the formulae for $S_{3}$ and $W_{5}$ in Section 3 . Now we study on $S_{4}$, namely, $\Sigma_{1,1,0, I}^{1,0}$ singularity.

Let $f:\left(\mathbf{C}^{n}, 0\right) \rightarrow T^{*} \mathbf{C}^{n}$ be an isotropic map-germ. Consider the ideal $\mathcal{J}_{1,1}^{1}$ in $\mathcal{O}_{n}$ generated by $n$-minors of the Jacobi matrix $\operatorname{Jac}(f)$ and $n$-minors of $\operatorname{Jac}(J(\pi \circ f), \pi \circ f)$.

LEMma 4.5. The ideal $\widetilde{\mathcal{J}}_{1,1}^{1}$ in $\mathcal{O}_{J_{I}^{2}}$ induced naturally from $\mathcal{J}_{1,1}^{1}$ defines the CohenMacaulay varieties $\overline{\Sigma_{1,1, I}^{1}}$ of codimension 3 in $J_{I}^{2} . \widehat{\mathcal{J}}$ is regular on the submanifold $\Sigma_{1,1, I}^{1}$

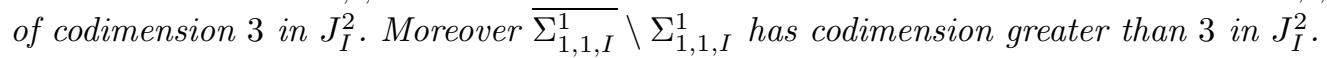


Proof. Since $\widehat{\mathcal{J}}_{1,1}^{1}$ defines $\overline{\Sigma_{1,1, I}^{1}}$, which a hypersurface in the submanifold $\Sigma_{I}^{1}$, we see $\overline{\Sigma_{1,1, I}^{1}}$ with the ideal $\widehat{\mathcal{J}}_{1,1}^{1}$ is a Cohen-Macaulay variety. Note that $\operatorname{codim}\left(\Sigma_{1,1, I}^{1}\right)=3$. For the typical singularity $S_{4}, f:\left(\mathbf{C}^{3}, 0\right) \rightarrow T^{*} \mathbf{C}^{3}$, we have $\operatorname{dim}_{\mathbf{C}} \mathcal{O}_{3} / \mathcal{J}_{1,1}^{1}=1$ by a simple calculation. This shows that $\widehat{\mathcal{J}}_{1,1}^{1}$ is regular on $\Sigma_{1,1, I}^{1}$. Since

$$
\overline{\Sigma_{1,1, I}^{1}} \backslash \Sigma_{1,1, I}^{1}=\overline{\Sigma_{2, I}^{1}}
$$

is of codimension 4, we have the result.

In particular we have the following formula, by Lemma 4.5, and by counting the intersection number of $j^{2} f\left(\mathbf{C}^{3}\right)$ and $\overline{\Sigma_{1,1, I}^{1}}\left(\mathbf{C}^{3}, T^{*} \mathbf{C}^{3}\right)$ in $J_{I}^{2}=J_{I}^{2}\left(\mathbf{C}^{3}, T^{*} \mathbf{C}^{3}\right)$ :

TheOREM 4.6. Let $f:\left(\mathbf{C}^{3}, 0\right) \rightarrow T^{*} \mathbf{C}^{3}$ be an isotropic map-germ of corank 1 . Then the number of $S_{4}$-singularities of a Lagrangian stable perturbation of $f$ is given by

$$
\# S_{4}=\operatorname{dim}_{\mathbf{C}} \mathcal{O}_{3} / \mathcal{J}_{1,1}^{1}
$$

provided $\operatorname{dim}_{\mathbf{C}} \mathcal{O}_{3} / \mathcal{J}_{1,1}^{1}<\infty$.

Lastly we turn to $T_{5}$ and $S_{5}$. Actually we study $\Sigma_{1,1,1,0, I}^{1,0}$ and $\Sigma_{2,0, I}^{1,0}$ singularities.

Let $f:\left(\mathbf{C}^{n}, 0\right) \rightarrow T^{*} \mathbf{C}^{n}$ be an isotropic map-germ of corank not greater than 1 . Consider the ideal $\mathcal{J}_{1,1,1}^{1}$ in $\mathcal{O}_{n}$ generated by $n$-minors of the Jacobi matrix $\operatorname{Jac}(f), n$-minors $J_{1}^{(2)}, \ldots, J_{n}^{(2)}$ (and $J(\pi \circ f)$ ) of $\operatorname{Jac}(J(\pi \circ f), \pi \circ f)$, and $n$-minors of $\operatorname{Jac}\left(J_{1}^{(2)}, \ldots, J_{n}^{(2)}, J(\pi \circ\right.$ $f), \pi \circ f)$.

Moreover consider the ideal $\mathcal{J}_{2}^{1}$ in $\mathcal{O}_{n}$ generated by $n$-minors of the Jacobi matrix $\operatorname{Jac}(f)$ and $(n-1)$-minors of $\operatorname{Jac}(\pi \circ f)$.

Then we have:

LEMMA 4.7.

(1) The ideal $\widetilde{\mathcal{J}}_{2}^{1}$ in $\mathcal{O}_{J_{I}^{1}}$ induced naturally from $\mathcal{J}_{2}^{1}$ defines the Cohen-Macaulay varieties $\overline{\Sigma_{2, I}^{1}}$ of codimension 4 in $J_{I}^{2} . \widehat{\mathcal{J}}_{2}^{1}$ is reduced on the submanifold $\Sigma_{2, I}^{1}$ of codimension 4 in $J_{I}^{1}$. Moreover $\overline{\Sigma_{2, I}^{1}} \backslash \Sigma_{2, I}^{1}$ has codimension greater than 4 in $J_{I}^{1}$.

(2) The ideal $\widehat{\mathcal{J}}_{1,1,1}^{1}$ in $\mathcal{O}_{J_{I}^{3}}$ induced naturally from $\mathcal{J}_{1,1,1}^{1}$ defines the variety

$$
\overline{\Sigma_{1,1,1, I}^{1} \cup \Sigma_{2, I}^{1}}
$$

of codimension 4 in $J_{I}^{3}$. $\widehat{\mathcal{J}}_{1,1,1}^{1}$ is reduced on the submanifold $\Sigma_{1,1,1, I}^{1,0}$ of codimension 4 in $J_{I}^{3}$, but of multiplicity 4 on the submanifold $\Sigma_{2,0 I}^{1,0}$. Moreover

$$
\overline{\Sigma_{1,1,1, I}^{1} \cup \Sigma_{2, I}^{1}} \backslash\left(\Sigma_{1,1,1, I}^{1,0} \cup \Sigma_{2,0, I}^{1,0}\right)
$$

has codimension greater than 5 in $J_{I}^{3}$.

Proof. (1) Set $X:=\overline{\Sigma_{2, I}^{1}} \subset J_{I}^{1}$, and $Y:=\Sigma_{I}^{1} \subset J_{I}^{1}$. Then $X$ has codimension 4 in $J_{I}^{1}$ ([15]). Moreover $X \subset Y \subset J_{I}^{1}$ and $Y$ is a regular submanifold of codimension 2 in $J_{I}^{1}$ (Section 3). Now let $z \in Y$. Then the $2 n$-column vectors of the $n \times 2 n$-matrix $z$ generate an $(n-1)$-dimensional vector space $V=V(z) \subset \mathbf{C}^{n}$ since $z \in \Sigma^{1}$. Take a local frame $\mathbf{e}_{1}, \ldots, \mathbf{e}_{n-1}$ near $z$ of $V$. Let $\mathbf{q}_{1}, \ldots, \mathbf{q}_{n}$ be the column vectors of $\operatorname{Jac}(\pi \circ z)$. Set

$$
\left(\mathbf{q}_{1}, \ldots, \mathbf{q}_{n}\right)=\left(\mathbf{e}_{1}, \ldots, \mathbf{e}_{n-1}\right) A(z) .
$$


Then $A(z)$ is a matrix of size $(n-1) \times n$ with entries in $\mathcal{O}_{Y}$. Then $z \in \overline{\pi^{-1} \Sigma_{2}}$ if and only if rank $A(z) \leq n-2$. We see in $\mathcal{O}_{Y}, \widehat{\mathcal{J}}_{2}^{1}$ agrees with the ideal generated by $(n-1)$-minors of $A(z)$. Moreover $X$ is of codimension 2 in $Y$. Thus $X$ is a determinantal variety in $Y$. Therefore $X$ is Cohen-Macaulay. For $T_{5}$ singularity, by a simple calculation, we have $\operatorname{dim}_{\mathbf{C}} \mathcal{O}_{4} / \mathcal{J}_{2}^{1}=1$.

(2) First note that the zero locus $Z=Z\left(\widehat{\mathcal{J}}_{1,1,1}^{1}\right)$ is included in $\Sigma_{I}^{1}$. Since $\widehat{\mathcal{J}}_{1,1}^{1}$ defines a hypersurface in $\Sigma_{I}^{1}$, we see there exists an element $J^{(2)} \in \mathcal{O}_{J_{I}^{3}}$ such that $\widehat{\mathcal{J}}_{1,1}^{1}=\widehat{\mathcal{J}}^{1}+$ $\left\langle J^{(2)}\right\rangle_{\mathcal{O}_{J_{I}^{3}}}$. Let $z \in \Sigma_{I}^{1}$. Then $n$-column vectors of $\operatorname{Jac}(f)$ generate an $(n-1)$-dimensional space $V=V(z)$. We take a local frame $\mathbf{e}_{1}, \ldots, \mathbf{e}_{n-1}$ of $V(z)$ over $\Sigma_{I}^{1} \subset J_{I}^{3}$. We add $\mathbf{e}_{n}$ to get a local frame $\mathbf{e}_{1}, \ldots, \mathbf{e}_{n-1}, \mathbf{e}_{n}$ of $\mathbf{C}^{n}$. We set

$$
\operatorname{Jac}\left(\pi \circ f, J(\pi \circ f), J^{(2)}\right)=\left(\mathbf{e}_{1}, \ldots, \mathbf{e}_{n-1}, \mathbf{e}_{n}\right) C(z),
$$

for an $n \times(n+2)$-times matrix $C(z)$. Then $\widehat{\mathcal{J}}_{1,1,1}^{1}$ is generated by $n$-minors of $C(z)$ over $\Sigma_{I}^{1}$. Thus we see

and it is of codimension 2 in $\Sigma_{I}^{1}$.

$$
Z\left(\widehat{\mathcal{J}}_{1,1,1}^{1}\right)=\overline{\Sigma_{1,1,1, I}^{1} \cup \Sigma_{2, I}^{1}}
$$

By a simple calculation, when $n=4$, we have $\operatorname{dim}_{\mathbf{C}} \mathcal{O}_{4} / \mathcal{J}_{1,1,1}^{1}=1$, for a $S_{5}$ singularity, and $\operatorname{dim}_{\mathbf{C}} \mathcal{O}_{4} / \mathcal{J}_{1,1,1}^{1}=4$, for a $T_{5}$ singularity. Here we present the calculation for $T_{5}$. For an isotropic map-germ $f:\left(\mathbf{C}^{n}, 0\right) \rightarrow T^{*} \mathbf{C}^{n}(n \geq 4)$ of type $T_{5}$, up to Lagrange equivalence, we have

$$
\pi \circ f\left(t, x_{2}, x_{3}, \ldots, x_{x-1}\right)=\left(\frac{1}{2} t^{2}-x_{2} x_{3},-\frac{1}{3} t^{3}-3 x_{2}^{2}-x_{2} x_{3} t-2 x_{2} x_{4}, x_{3}, \ldots, x_{n-1}\right),
$$

and moreover, up to a symplectomorphism of $\left(T^{*} \mathbf{C}^{n}, f(0)\right)$, we have

$$
f\left(t, x_{2}, x_{3}, \ldots, x_{x-1}\right)=\left(x_{2} t, \frac{1}{3} t^{3}, 0, \ldots, 0, \frac{1}{2} t^{2}, x_{2}, x_{3}, \ldots, x_{n-1}\right) .
$$

Note that a diffeomorphism of $\left(T^{*} \mathbf{C}^{n}, f(0)\right)$ preserves the ideal $\mathcal{J}^{1}$ generated by $n$-minor of $\operatorname{Jac}(f)$. Then we have

$$
\mathcal{J}_{1,1,1}^{1}=\left\langle t, x_{2}, x_{3} x_{4}, x_{4}^{2}, x_{3}^{3}\right\rangle_{\mathcal{O}_{n}},
$$

which defines 4 -fold $T_{5}$-locus $\left\{t=0, x_{2}=0, x_{3}=0, x_{4}=0\right\}$. Thus we have the required results.

By Lemma 4.7, counting the intersection number of $j^{3} f\left(\mathbf{C}^{4}\right)$ with the zero locus $Z\left(\widehat{\mathcal{J}}_{2}^{1}\right)$ and $Z\left(\widehat{\mathcal{J}}_{1,1,1}^{1}\right)$ in $J_{I}^{3}\left(\mathbf{C}^{4}, T^{*} \mathbf{C}^{4}\right)$, we have:

TheOREM 4.8. Let $f:\left(\mathbf{C}^{4}, 0\right) \rightarrow T^{*} \mathbf{C}^{4}$ be an isotropic map-germ of corank not greater than 1 . Then the number $\# T_{5}$ of $T_{5}$-singularities of a Lagrangian stable perturbation of $f$ is given by the following formula:

$$
\# T_{5}=\operatorname{dim}_{\mathbf{C}} \mathcal{O}_{4} / \mathcal{J}_{2}^{1},
$$

provided $\operatorname{dim}_{\mathbf{C}} \mathcal{O}_{4} / \mathcal{J}_{2}^{1}<\infty$.

Proposition 4.9. Let $f:\left(\mathbf{C}^{4}, 0\right) \rightarrow T^{*} \mathbf{C}^{4}$ be an isotropic map-germ of corank not exceeding 1 . Then the number $\# S_{5}$ of $S_{5}$-singularities is estimated by

$$
\# S_{5} \leq \operatorname{dim}_{\mathbf{C}} \mathcal{O}_{4} / \mathcal{J}_{1,1,1}^{1}-4 \operatorname{dim}_{\mathbf{C}} \mathcal{O}_{4} / \mathcal{J}_{2}^{1},
$$

provided $\operatorname{dim}_{\mathbf{C}} \mathcal{O}_{4} / \mathcal{J}_{2}^{1}<\infty$ and $\operatorname{dim}_{\mathbf{C}} \mathcal{O}_{4} / \mathcal{J}_{1,1,1}^{1}<\infty$. 
5. Open questions. We collect here several practical questions related to the results in this paper.

(Q1) Recall that the formulae for the numbers of $A_{4}$ and of $D_{4}$ for Lagrangian immersions are given in Section 2. Thus we ask: Do the same formulae hold on the numbers of $A_{4}$ and $D_{4}$, not only for a Lagrange immersion, but also for an isotropic map-germ of corank one $\left(\mathbf{C}^{3}, 0\right) \rightarrow T^{*} \mathbf{C}^{3}$ ?

(Q2) We have observed a similarity between the pair $\left(D_{4}, A_{4}\right)$ and the pair $\left(T_{5}, S_{5}\right)$ in Section 4. Does the similarity have any reason?

(Q3) What are the formulae for the number of $A_{5}$ and $D_{5}$ of a Lagrangian immersion and of an isotropic map-germ of corank one? See Remark 2.6.

(Q4) We can formulate the following conjecture:

Let $f:\left(\mathbf{C}^{n}, 0\right) \rightarrow T \mathbf{C}^{n}$ be an isotropic map-germ of corank at most one. Then the number $\# \Sigma_{i_{1}, i_{2}, \ldots, I}^{1_{k}}$ of isolated singularities, with an isotropic Thom-Boardman symbol $\Sigma_{i_{1}, i_{2}, \ldots, I}^{1_{k}}$, appearing in a Lagrange stable perturbation of $f$ is equal to the dimension of the quotient $\mathcal{O}_{n} / \mathcal{J}_{i_{1}, i_{2}, \ldots}^{1_{k}}$ by the Morin ideal $\mathcal{J}_{i_{1}, i_{2}, \ldots}^{1_{k}}$ for the corresponding (non-isotropic) Thom-Boardman singularity $\Sigma_{i_{1}, i_{2}, \ldots}^{1_{k}}$ of composed mappings.

Is the conjecture true in general? We have shown in this paper, it is true for the cases $A_{2}, A_{3}, S_{3}, S_{4}, T_{5}$ and for $W_{5}$, in the nice range $n \leq 4$, except for the cases $A_{4}, D_{4}, A_{5}$ and $S_{5}$. Remark that $D_{5}$-singularity is not described as a Thom-Boardman singularity (Remark 2.6). See Sections 2, 3 and 4 for the details.

\section{References}

[1] V. I. Arnol'd, S. M. Guseŭn-Zade, A. N. Varchenko, Singularities of Differentiable Maps I, Monogr. Math. 82, Birkhäuser, Boston, 1985.

[2] I. A. Bogaevskii, G. Ishikawa, Lagrange mappings of the first open Whitney umbrella, Pacific J. Math. 203 (2002), 115-138.

[3] T. Fukuda, G. Ishikawa, On the number of cusps of stable perturbations of a plane-to-plane singularity, Tokyo J. Math. 10 (1987), 375-384.

[4] T. Fukui, J. J. Nuño Ballesteros, M. J. Saia, On the number of singularities in generic deformations of map germs, J. London Math. Soc. (2) 58 (1998), 141-152.

[5] T. Fukui, J. Weyman, Cohen-Macaulay properties of Thom-Boardman strata. I. Morin's ideal, Proc. London Math. Soc. (3) 80 (2000), 257-303.

[6] T. Fukui, J. Weyman, Cohen-Macaulay properties of Thom-Boardman strata. II. The defining ideals of $\Sigma^{i, j}$, preprint.

[7] W. Fulton, Intersection Theory, Ergeb. Math. Grenzgeb. (3) 2, Springer, Berlin, 1984.

[8] T. Gaffney, D. M. Q. Mond, Cusps and double folds of germs of analytic maps $\mathbf{C}^{2} \rightarrow \mathbf{C}^{2}$, J. London Math. Soc. (2) 43 (1991), 185-192.

[9] A. B. Givental', Lagrangian imbeddings of surfaces and open Whitney umbrella, Funktsional. Anal. i Prilozhen. 20-3 (1986), 35-41 (Russian); English transl.: Funct. Anal. Appl. 20 (1986), 197-203.

[10] A. B. Givental', Singular Lagrangian manifolds and their Lagrangian mappings, Itogi Nauki Tekhniki, Sovrem. Probl. Mat. Nov. Dost. 33, VINITI, Moskva, 1988, 55-112 (Russian); English transl.: J. Soviet Math. 52 (1990), 3246-3278. 
[11] G. M. Greuel, Die Zahl der Spitzen und die Jacobi-Algebra einer isolierten Hyperflächensingularität, Manuscripta Math. 21 (1977), 227-241.

[12] G. M. Greuel, Ergänzung und Berichtigung zu: "Die Zahl der Spitzen und die Jacobi-Algebra einer isolierten Hyperflächensingularität", Manuscripta Math. 25 (1978), 205-208.

[13] M. Hochster, J. A. Eagon, Cohen-Macaulay rings, invariant theory, and the generic perfection of determinantal loci, Amer. J. Math. 93 (1971), 1020-1058.

[14] G. Ishikawa, The local model of an isotropic map-germ arising from one-dimensional symplectic reduction, Math. Proc. Cambridge Philos. Soc. 111 (1992), 103-112.

[15] G. Ishikawa, Transversalities for Lagrange singularities of isotropic mappings of corank one, in: Singularities and Differential Equations, Banach Center Publ. 33, Warszawa, 1996, 93-104.

[16] G. Ishikawa, Symplectic and Lagrange stabilities of open Whitney umbrellas, Invent. Math. 126 (1996), 215-234.

[17] G. Ishikawa, Determinacy, transversality and Lagrange stability, in: Geometry and Topology of Caustics - Caustics '98, Banach Center Publ. 50, Warszawa, 1999, 123-135.

[18] B. Iversen, Lê Dũng Tráng, Calcul du nombre de cusps dans la déformation semi-universelle d'une singularité isolée d'hypersurface complexe, Bull. Soc. Math. France 102 (1974), 99-107.

[19] W. L. Marar, D. Mond, Multiple point schemes for corank 1 maps, J. London Math. Soc. (2) 39 (1989), 553-567.

[20] W. L. Marar, J. A. Montaldi, M. A. S. Ruas, Multiplicities of zero-schemes in quasihomogeneous corank-1 singularities $\mathbf{C}^{n} \rightarrow \mathbf{C}^{n}$, in: Singularity Theory (Liverpool, 1996), London Math. Soc. Lecture Note Ser. 263, Cambridge Univ. Press, Cambridge, 1999, 353-367.

[21] H. Matsumura, Commutative Algebra, second ed., Math. Lecture Note Ser. 56, Benjamin, Reading, 1980.

[22] D. Mond, Some remarks on the geometry and classification of germs of maps from surfaces to 3-space, Topology 26 (1987), 361-383.

[23] D. Mond, Vanishing cycles for analytic maps, in: Singularity Theory and its Applications, Part I, Lecture Notes in Math. 1462, Springer, Berlin, 1991.

[24] D. Mond, The number of vanishing cycles for a quasihomogeneous mapping from $\mathbf{C}^{2}$ to $\mathbf{C}^{3}$, Quart. J. Math. Oxford Ser. (2) 42 (1991), 335-345.

[25] B. Morin, Calcul jacobien, Ann. Sci. École Norm. Sup. (4) 8 (1975), 1-98.

[26] J. J. Nuño Ballesteros, M. J. Saia, Multiplicity of Boardman strata and deformations of map germs, Glasgow Math. J. 40 (1998), 21-32.

[27] F. Pham, Courbes discriminantes des singularités planes d'ordre 3, in: Singularités à Cargèse, Astérisque 7/8, Soc. Math. France, Paris, 1973, 363-391.

[28] J. H. Rieger, Families of maps from the plane to the plane, J. London Math. Soc. (2) 36 (1987), 351-369.

[29] J. H. Rieger, Versal topological stratification and the bifurcation geometry of map-germs of the plane, Math. Proc. Cambridge Philos. Soc. 107 (1990), 127-147.

[30] V. M. Zakalyukin, Reconstructions of fronts and caustics depending on a parameter, and versality of mappings, Itogi Nauki Tekhniki, Sovrem. Probl. Mat. 22, VINITI, Moskva, 1983, 56-93 (Russian); English transl.: J. Soviet Math. 27 (1984), 2713-2735.

[31] V. M. Zakalyukin, Generating ideals of Lagrangian varieties, in: Theory of Singularities and its Applications, Adv. Soviet Math. 1, Amer. Math. Soc., Providence, 1990, 201-210. 